
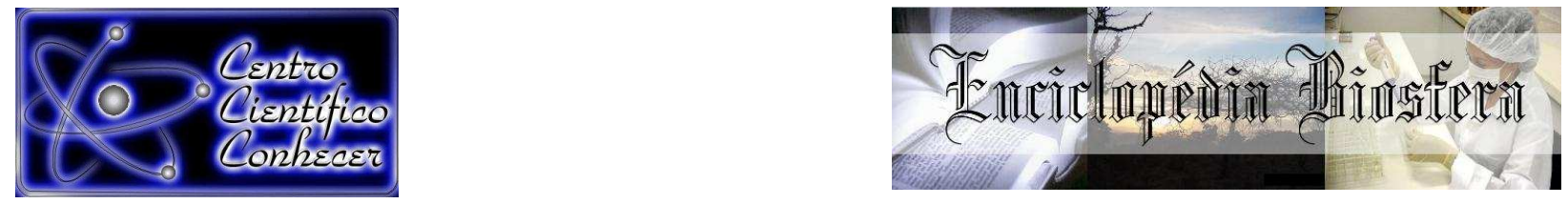

\title{
GERMINAÇÃO DE SEMENTES DE FEIJÃO-CAUPI SOB DIFERENTES PROPORÇÕES DE CAMA DE FRANGO E PALHA DE CAFÉ
}

Tatiane Melo de Lima1, Fábio Janoni Carvalho², Marcela Ribeiro Violatti², Elias Antônio Borges ${ }^{3}$, Vitor D’ Alessandro Langoni ${ }^{3}$.

${ }^{1}$ Prof. Msc. da Universidade Federal de Uberlândia, Monte Carmelo-Minas Gerais

${ }^{2}$ Eng. Agr., Mestrando em Fitotecnia da Universidade Federal de Uberlândia (fabiojanoni@ufu.br), Uberlândia-Minas Gerais

${ }^{3}$ Graduando em Agronomia da Universidade Federal de Uberlândia, Monte Carmelo Minas Gerais

Recebido em: 08/09/2015 - Aprovado em: 14/11/2015 - Publicado em: 01/12/2015 DOI: http://dx.doi.org/10.18677/Enciclopedia_Biosfera_2015_167

\begin{abstract}
RESUMO
O feijão-caupi é uma das principais culturas de subsistência do Norte e Nordeste do Brasil onde compostos orgânicos surgem como alternativa para adubação. Diante disso, objetivou-se verificar a interferência das diferentes combinações dos compostos cama de frango e palha de café na germinação de sementes do feijãocaupi subclasse Vinagre. $O$ experimento foi realizado em casa de vegetação da Universidade Federal de Uberlândia em Monte Carmelo. Foram utilizadas seis misturas entre cama de frango, palha de café e solo. O solo foi peneirado e os resíduos orgânicos foram adicionados seguindo as seguintes proporções (solo cama de frango - palha de café): 3-1-1, 3-2-1, 3-1-2, 3-0-1, 3-1-0, 3-0-0 e testemunha com fertilizante mineral, totalizando sete tratamentos. $O$ delineamento utilizado foi o de blocos casualizados com três blocos e 21 parcelas experimentais. As misturas foram colocadas em vasos de 10 litros e a semeadura foi realizada com 20 sementes por parcela. A semeadura ocorreu no dia 29/05/2015 e as contagens da germinação feitas diariamente. Os dados foram submetidos à análise de variância (teste F) após o atendimento das pressuposições do modelo pelos testes de Shapiro-Wilk, para normalidade dos resíduos, e de Levene, para homogeneidade das variâncias. As médias foram comparadas pelo teste de Tukey, a $5 \%$ de probabilidade. Dos caracteres germinativos apenas $0 \mathrm{CV}_{t}$ foi igual em todas as misturas. Os tratamentos 3-0-1 e 3-0-0 apresentaram os demais caracteres germinativos superiores. Já as misturas 3-2-1, 3-1-2 e 3-1-1 evidenciaram efeito tóxico.
\end{abstract}

PALAVRAS-CHAVE: agricultura familiar, Resíduos orgânicos, velocidade de emergência, Vigna unguiculata.

\section{COWPEA SEED GERMINATION IN DIFFERENT PROPORTIONS OF POULTRY LITTER AND COFFEE STRAW}

\begin{abstract}
Cowpea is a leading North and Northeastern subsistence crop in Brazil where organic compounds are an alternative of fertilizer. The objective of this research was
\end{abstract}


to verify the interference of different combinations of poultry litter and coffee straw compounds on germination of cowpea seeds subclass Vinagre. The experiment was conducted in a greenhouse at the Universidade Federal de Uberlândia in Monte Carmelo. Six mixtures with poultry litter, coffee straw and soil were used. The soil was sifted and organic compounds were added according to the following proportions (soil - poultry litter - coffee straw): 3-1-1, 3-2-1, 3-1-2, 3-0-1, 3-1-0, 3-0-0 and control with mineral fertilizer, totaling seven treatments. The design was a randomized complete block design with three blocks and 21 plots. The mixtures were placed in 10 liter pots and seeds were sown with 20 seeds per plot. Sowing was made on 05/29/2015 and scores of germination were noted daily. Data were subjected to analysis of variance (F's test) after attempted the assumptions of the model by Shapiro-Wilk test for normal residuals, and Levene for homogeneity of variances. The means were compared by Tukey test at $5 \%$ probability. Of all germination characters, only $C V_{t}$ was the same in all mixtures. Treatments 3-0-1 and 3-0-0 had higher germinations. However mixtures 3-2-1, 3-1-2 and 3-1-1 showed toxic effect.

KEYWORDS: Organic residues, emergency speed, Vigna unguiculata, family farming.

\section{INTRODUÇÃO}

O feijoeiro Vigna [Vigna unguiculata (L.) Walp], pertencente a família Fabaceae, conhecido como feijão-caupi, feijão de corda ou feijão macassar, destaca-se entre as principais culturas de subsistência nas regiões Norte e Nordeste do Brasil. É uma leguminosa benéfica à alimentação humana devido ao alto teor de proteínas e aminoácidos, muito presente na dieta alimentar da população que reside no Sertão Nordestino. A cultura é também utilizada como fonte de diversificação de renda, principalmente em pequenas propriedades (SILVA et al., 2013).

Devido à geração de empregos diretos e indiretos e a quantidade de mão de obra demandada em seu cultivo, tornou-se uma cultura de grande relevância socioeconômica (SALGADO et al., 2012). Os produtores são principalmente de pequeno ou médio porte, com intuito de subsistência e venda da produção excedente.

Geralmente pequenos produtores não possuem recurso financeiro para investir em tecnologias de aplicação, produtos químicos, adubação do solo, entre outros fatores que auxiliam para maior produção e lucratividade (SILVA et al., 2014). Formas alternativas de produção agrícola são cada vez mais difundidas, devido aos baixos custos de implantação, menores danos ao meio ambiente, e incremento na produção que as mesmas oferecem. Segundo PEREIRA et al. (2013), a utilização de compostos orgânicos, a fim de substituir ou complementar a adubação química apresentam diversos benefícios, entre eles: baixo custo, aproveitamento de resíduos, manutenção da produtividade, melhoria da estrutura e fertilidade dos solos e menor utilização de fertilizantes químicos solúveis.

Os fertilizantes orgânicos, além de disponibilizarem nutrientes, melhoram os teores de matéria orgânica e contribuem para melhor estrutura do solo, aumentando a capacidade de retenção de umidade, infiltração da água da chuva, atividade de microrganismos e troca catiônica. A cama de frango e palha de café se tornaram opções viáveis por serem excelentes fontes nutricionais, e quando manejados de forma adequada, conseguem suprir as necessidades da cultura, parcial ou totalmente (GUARESCHI et al., 2013). 
A composição mineral do composto cama de frango apresenta frações com solubilidades diversas, algumas prontamente disponíveis às plantas e outras na forma orgânica, que dependem da atividade biológica do solo para serem mineralizadas. Para o nitrogênio, estima-se que 60 \% estejam na forma orgânica, 30 \% como amônio e 10 \% na forma nítrica e amídica (SIMS \& WOLF, 1994). Palhas de café provenientes do beneficiamento do fruto podem constituir uma boa fonte de potássio, que, por meio de ação hídrica, pode proporcionar aumento dos teores do nutriente no solo (ZOCA et al., 2014).

Apesar da importância do uso destes compostos orgânicos, principalmente pelo pequeno produtor, ainda não há estudos que visam compreender sua interferência na germinação do feijão-caupi, pois os compostos liberados podem tanto favorecer quanto dificultar a germinação. Diante disso, o objetivo do trabalho foi verificar se as diferentes combinações dos compostos cama de frango e palha de café interferem na germinação das sementes do feijão-caupi subclasse Vinagre.

\section{MATERIAL E MÉTODOS}

O experimento foi realizado em casa de vegetação da Universidade Federal de Uberlândia Campus Monte Carmelo (1842'43,19"S e 4729'55,8"O). Foram utilizadas seis misturas entre cama de frango, palha de café e solo. Para a mistura, foi utilizado Latossolo vermelho proveniente de barranco, típico da região do Cerrado. Os resíduos da cama de frango e palha de café foram obtidos de fazendas próximas à região e então curtidos para seu uso.

O solo foi peneirado e os resíduos orgânicos foram adicionados seguindo as seguintes proporções (solo - cama de frango - palha de café): 3-1-1, 3-2-1, 3-1-2, 30-1, 3-1-0, 3-0-0 e testemunha com fertilizante mineral, totalizando sete tratamentos. O delineamento utilizado foi o de blocos casualizados com três blocos, totalizando 21 parcelas experimentais. Para a testemunha com fertilizante mineral foi adicionado em cada vaso o equivalente a $60 \mathrm{~kg} \mathrm{~N} \mathrm{ha}^{-1}, 60 \mathrm{~kg} \mathrm{P}_{2} \mathrm{O}_{5} \mathrm{ha}^{-1}$ e $60 \mathrm{~kg} \mathrm{~K}_{2} \mathrm{O}$ ha ${ }^{-1}$, conforme indicação da Embrapa para a cultura (FILHO et al., 2003), com a utilização de ureia, cloreto de potássio e superfosfato simples. A adubação foi feita no sulco da semeadura, porém sem o contato direto da semente com o adubo.

As misturas foram colocadas em vasos de 10 litros e a semeadura foi realizada com 20 sementes de feijão-caupi subclasse Vinagre por parcela. A irrigação foi constante, mantendo os vasos sempre com a capacidade de campo acima de $60 \%$. A semeadura ocorreu no dia 29 de maio de 2015 e as contagens da germinação foram diárias com o intuito de calcular o tempo inicial ( $t_{\xi}$, final $\left(t_{f}\right)$ e médio de germinação $(\bar{t})$ germinação $(G)$; velocidade média $(\bar{v})$ (LABOURIAU, 1983); coeficiente de variação do tempo ( $C V_{k}$ (RANAL \& SANTANA, 2006); velocidade de germinação $(V G)$ e frequência relativa de germinação $\left(f_{i}\right)$ (LABOURIAU \& VALADARES, 1976).

O tempo inicial consistiu do dia em que a primeira semente do vaso germinou e, o tempo final, o dia da última germinação. O tempo médio de germinação $(\bar{t})$ foi calculado pela fórmula: $\bar{t}=\sum_{i=1}^{k} n_{i} t_{i} / \sum_{i=1}^{k} n_{i}$; em que $t_{i}$ é o tempo entre o início do experimento e a i-ésima observação; $n_{i}$ é o número de sementes germinadas no tempo $i$ (não acumulado); e $k$ a última germinação.

Utilizou-se da expressão $\bar{v}=1 / \bar{t}$, para cálculo da velocidade média e $V E=\sum_{i=1}^{k} n_{i} / t_{i}$ para a velocidade de germinação. $O$ coeficiente de variação do 
tempo $\left(C V_{t}\right)$ foi calculado pela expressão $C V_{t}=\left(s_{t} / t\right) 100$; onde $s_{t}$ é o desvio padrão do tempo e $\bar{t}$ o tempo médio de germinação. A frequência relativa de germinação $\left(f_{i}\right)$ foi calculada como $f_{i}=n_{i} / \Sigma_{i=1}^{k} n_{i}$.

Os dados obtidos foram submetidos à análise de variância (teste F) após o atendimento das pressuposições do modelo pelos testes de Shapiro-Wilk, para normalidade dos resíduos, e de Levene, para homogeneidade das variâncias, ambos a $1 \%$ de significância. As médias foram comparadas pelo teste de Tukey, a $5 \%$ de probabilidade.

\section{RESULTADOS E DISCUSSÃO}

Os dados referentes aos caracteres germinativos da cultivar foram submetidos à análise de variância (Tabela 1). Dentre as variáveis analisadas, somente o coeficiente de variação do tempo $(C V)$ não foi influenciado de forma significativa pelas diferentes misturas. Segundo RANAL \& SANTANA (2006), o coeficiente de variação do tempo mede o grau de dispersão da germinação ao redor do tempo médio. Assim, os resultados demonstram que a germinação foi concentrada próxima ao tempo médio, com valores de até 15,72\% (Tabela 2), revelando que o período de germinação da espécie em geral não é longo.

TABELA 1. Resumo da análise de variância dos caracteres de germinação de feijão caupi subclasse Vinagre sob as diferentes misturas de solo e resíduos orgânicos.

\begin{tabular}{cccccccc}
\hline & $t_{i}$ & $t_{f}$ & $\bar{t}$ & $\mathrm{G}$ & $\bar{v}$ & $C V_{t}$ & $V E$ \\
\hline $\mathrm{QM}$ & 0,714 & 1,302 & 1,804 & 630,159 & 0,001 & 2,777 & 1,928 \\
$\mathrm{QM}_{\text {erro }}$ & 0,143 & 0,111 & 0,118 & 80,754 & 0,000 & 7,715 & 0,130 \\
$\mathrm{~F}$ & 5,000 & 11,714 & 15,278 & 7,803 & 16,963 & 2,777 & 14,874 \\
p-valor & $0,009^{* *}$ & $0,000^{* *}$ & $0,000^{* *}$ & $0,001^{* *}$ & $0,000^{* *}$ & 0,062 & $0,000^{* *}$
\end{tabular}

${ }^{\star *}$ Significativo a $1 \%$ pelo teste de F. QM: Quadrado Mé Última Germinação, :Tempo Médio para a Germinação, G: Ġerminação, : Velocidade Média; cvt: Coeficiente de Variação do Tempo, VE: Velocidade De Emergência, CV: Coeficiente de Variação.

Em relação aos demais caracteres, os tratamentos 3-0-1 e 3-0-0 apresentaram padrões germinativos superiores pelo teste de Tukey a 0,05 de significância (Tabela 2), com menores tempos inicial, médio e final, mostrando germinação antecipada. A taxa de germinação, velocidade média e velocidade de emergência em ambos os tratamentos foi de $97 \%, 1,9 \mathrm{sem}$. dia ${ }^{-1}$ e 3,7 sem.dia ${ }^{-1}$, respectivamente, indicando que a mistura com uma parte de palha de café (3-0-1) não demonstrou nenhuma redução nos caracteres germinativos.

Já as misturas com proporções maiores de cama de frango e palha de café, 3-2-1, 3-1-2 e 3-1-1, afetaram principalmente a taxa de germinação, que foram de 57,73 e $77 \%$ respectivamente, mostrando o efeito tóxico destas misturas. Resultados semelhantes foram descritos por BACILIO et al. (2003), no qual o esterco bovino gerou efeito inibitório sobre a germinação de sementes de trigo pelo seu alto nível de ácidos húmicos. 
TABELA 2. Médias dos caracteres germinativos do feijão caupi subclasse Vinagre submetido a diferentes misturas de solo e resíduos orgânicos.

\begin{tabular}{cccccccc}
\hline $\begin{array}{c}\text { Misturas } \\
\text { (S:CF:PC) }\end{array}$ & $t_{i}$ (dia) & $t_{f}$ (dia) & $\bar{t}$ (dia) & $\mathrm{G}(\%)$ & $\bar{v}\left(\right.$ dia $\left.^{-1}\right)$ & cvt $(\%)$ & $V E\left(\right.$ s.dia $\left.{ }^{1}\right)$ \\
\hline $3-1-1$ & $5,00 \mathrm{a}$ & $8,00 \mathrm{c}$ & $7,13 \mathrm{~b}$ & $76,67 \mathrm{ab}$ & $0,15 \mathrm{~b}$ & 15,72 & $2,35 \mathrm{bc}$ \\
$3-2-1$ & $6,33 \mathrm{~b}$ & $8,00 \mathrm{c}$ & $6,74 \mathrm{~b}$ & $56,67 \mathrm{~b}$ & $0,14 \mathrm{~b}$ & 8,23 & $1,61 \mathrm{c}$ \\
$3-1-2$ & $5,33 \mathrm{ab}$ & $8,00 \mathrm{c}$ & $6,52 \mathrm{~b}$ & $73,33 \mathrm{ab}$ & $0,15 \mathrm{~b}$ & 13,31 & $2,29 \mathrm{bc}$ \\
$3-0-1$ & $5,00 \mathrm{a}$ & $7,00 \mathrm{ab}$ & $5,35 \mathrm{a}$ & $96,67 \mathrm{a}$ & $0,19 \mathrm{a}$ & 12,54 & $3,67 \mathrm{a}$ \\
$3-1-0$ & $5,33 \mathrm{ab}$ & $7,67 \mathrm{bc}$ & $6,34 \mathrm{~b}$ & $86,97 \mathrm{a}$ & $0,16 \mathrm{~b}$ & 12,11 & $2,78 \mathrm{ab}$ \\
$3-0-0$ & $5,00 \mathrm{a}$ & $6,33 \mathrm{a}$ & $5,28 \mathrm{a}$ & $96,67 \mathrm{a}$ & $0,19 \mathrm{a}$ & 8,39 & $3,70 \mathrm{a}$ \\
Fert. Mineral & $5,00 \mathrm{a}$ & $7,00 \mathrm{ab}$ & $5,28 \mathrm{a}$ & $90,00 \mathrm{a}$ & $0,19 \mathrm{a}$ & 11,27 & $3,45 \mathrm{a}$ \\
\hline
\end{tabular}

Médias seguidas de letras iguais, na coluna, não diferem significamente a $5 \%$ de probabilidade pelo teste de Tukey. S: Solo, CF: Cama de Frango, PC: Palha de Café; $\boldsymbol{t}_{i}$ : tempo da primeira germinação, $t_{\text {: }}$ :

Tempo da Última Germinação, ĩ: Tempo Médio para a Germinação, G: Germinação, vi: Velocidade Média; cvt: Coeficiente de Variação do Tempo, VE: Velocidade de Emergência.

OLIVEIRA et al. (2001) observaram não haver diferenças na germinação de sementes do feijão-caupi cv. IPA-206 submetidas à adubação orgânica na presença e ausência de adubação mineral, cujo percentual máximo obtido foi de $88 \%$. ABEBE et al., (2005), relataram que a aplicação de doses de composto orgânico bovino em vasos na equivalência de 10 a $100 \mathrm{t} \mathrm{ha}^{-1}$ também não surtiram efeitos sobre a germinação de caupi.

Segundo MATA et al. (2011), a aplicação de doses acima de 20 toneladas de esterco bovino por hectare favorecem a germinação e o desenvolvimento de plântulas de milho. Logo, entende-se que o aumento de germinação pela adição de compostos orgânicos pode estar relacionada à espécie e aos nutrientes fornecidos. Resultados satisfatórios com utilização de esterco bovino e frango foram relatados por ADEOYE et al. (2011), devido ao aumento da área foliar, altura, diâmetro de caule e a produtividade de feijão-caupi, além de aumentar o teor de matéria orgânica e a condutividade elétrica do solo.

DUTRA et al. (2012) relataram efeito aditivo em sementes de feijão-caupi sob manejo da adubação com compostos orgânicos associada a fertilizantes minerais. Outros autores também encontraram resultados favoráveis para a utilização de adubo orgânico associado ao adubo mineral. Em feijão-caupi, OLIVEIRA et al. (2001) obtiveram rendimento de $3,03 \mathrm{t} \mathrm{ha}^{-1}$, pelo uso de esterco na presença de adubo mineral, e com sua ausência $2 \mathrm{t} \mathrm{ha}^{-1}$. GALBIATTI (2011) observou que os tratamentos que receberam doses crescentes de adubos orgânicos complementados com adubação mineral a produtividade foi superior quando comparado aos tratamentos que não receberam.

Segundo DUTRA et al. (2012) o índice de velocidade de emergência em plantas de feijão-caupi foi afetado pela adubação nitrogenada, com os maiores valores obtidos das sementes oriundas das plantas cultivadas sem adubação nitrogenada e adubadas com nitrogênio na fundação, independente do nível. Resultados de CRUSCIOL et al. (2003) também relatam no feijão de corda cv. IACCarioca, menores valores de velocidade de emergência em maiores níveis de nitrogênio, sendo estatisticamente inferior daquelas sementes oriundas das plantas não adubadas. Entretanto, para o experimento, nos tratamentos com a adubação de ureia não ocorreu interferência na germinação (90\%) e velocidade de emergência

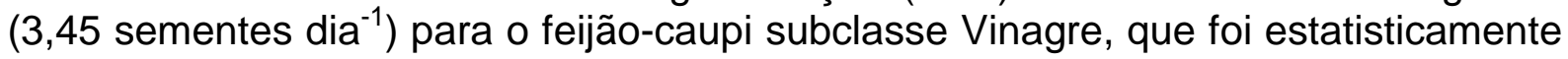
igual ao tratamento com apenas solo.

Atentando-se à frequência relativa da germinação, os tratamentos com as melhores frequências relativas ao longo do tempo foram com a mistura de três 
partes de solo para uma de palha de café e com apenas solo ou fertilizante mineral, com curvas mais acentuadas sinalizando germinações maiores e rápidas. Os padrões para a frequência destes tratamentos foram os mesmos encontrados por SOUZA et al. (2015) na germinação do feijão Serrano (Phaseolus vulgaris cv. Serrano). Além disso, a frequência de germinação está associada ao índice de sincronização da germinação (BUFALO et al., 2012), mostrando que a maiorias das misturas com os compostos orgânicos diminui a sincronia da germinação entre as sementes e acarreta em germinação dispersa, deixando as sementes mais propensas a possíveis intempéries.
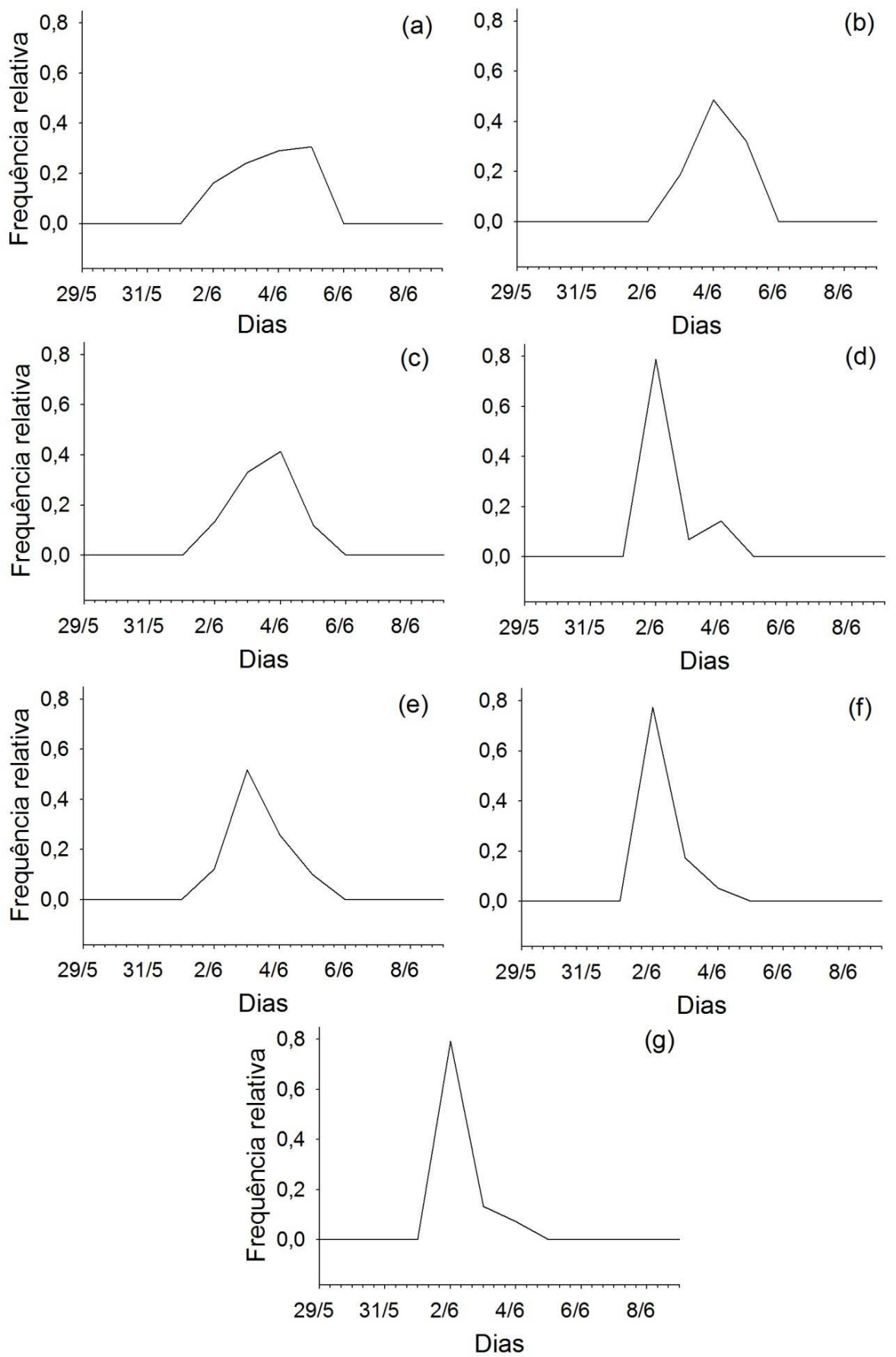

FIGURA 1. Gráfico da frequência relativa da germinação do feijão-caupi subclasse Vinagre sob diferentes misturas de composto na proporção solo- cama de frango-palha de café. (a) 3-1-1, (b) 3-2-1, (c) 3-1-2, (d) 3-0-1, (e) 3-1-0, (f) 3-0-0, (g) fertilizante mineral. 


\section{CONCLUSÃO}

A utilização da palha de café é uma alternativa viável quando houver grande disponibilidade e quantidade deste resíduo orgânico na propriedade rural, ou então, como uma alternativa ambientalmente correta de destinação final deste resíduo, evitando contaminação de corpos da água, nascentes e riachos, mas não em quantidades superiores de uma parte de café por três de solo. A cama de frango também pode ser utilizada, entretanto deve-se atentar que seu uso é mais tóxico para as sementes do feijão-caupi subclasse Vinagre, devendo o produtor aumentar a densidade de semeadura afim de obter o mesmo estande final.

\section{REFERÊNCIAS}

ABEBE, G., HATTAAR, B., AL-TAWAHA, A. Nutrient Availability as affected by manure application to cowpea (Vigna unguiculata L. Walp) on calcareous soils. Journal of Agriculture and Social Sciences. v.1, n.1, p.1-6, 2005.

ADEOYE, P.A.; ADEBAYO, S.E.; MUSA, J.J. Growth and response of cowpea (Vigna unguiculata) to poultry and cattle manure as amendments on sandy loam soil plot. Agricultural Soil, v.6, n.5, p. 218-221, 2011.

BACILIO, M. ; VAZQUEZ, P. ; BASHAN, Y. Alleviation of noxious effects of cattle ranch composts on wheat seed germination by inoculation with Azospirillum spp. Biology and Fertility of Soils, v.38, p. 261-266, 2003.

BUFALO J.; AMARO, A.C.E.; ARAÚJO, H. S. ;CORSATO, J. M.; ONO, E. O.; FERREIRA, G.; RODRIGUES, J. D. Períodos de estratificação na germinação de sementes de alface (Lactuca sativa L.) sob diferentes condições de luz e temperatura. Semina: Ciências Agrárias, v.33, n.3, p. 931-940, 2012.

CRUSCIOL, C.A.C.; LIMA, E.D.; ANDREOTTI, M.; NAKAGAWA, J.; LEMOS, L.B.; MARUBAYASHI, O.M. Efeito do nitrogênio sobre a qualidade fisiológica, produtividade e características de sementes de feijão. Revista Brasileira de Sementes, v.25, n.1, p. 108-115, 2003.

DUTRA, A.S.; BEZERRA, F.T.C.; NASCIMENTO, P.R.; LIMA, D.C. Produtividade e qualidade fisiológica de sementes de feijão caupi em função da adubação nitrogenada. Revista Ciência Agronômica, v. 43, n. 4, p. 816-821, 2012.

FILHO, M.P.B.; FAGÉRIA, N.K.; SILVA, O.F. Cultivo do Feijoeiro Comum. Sistemas de Produção 2, Embrapa Arroz e Feijão, 2003. Versão eletrônica. Disponível em: http://sistemasdeproducao.cnptia.embrapa.br/ Acesso em: 10/08/2015.

GALBIATTI, J.A.; SILVA, F.G.; FRANCO, C.F.; CARAMELO, A.D. Desenvolvimento do feijoeiro sob o uso de biofertilizante e adubação mineral. Engenharia Agrícola, Jaboticabal, v.31, n.1, p.167-177, 2011. 
GUARESCHI, R.; PERIN, A.; ANDRADE, D.; ROCHA, A. Adubação com cama de frango e esterco bovino na produtividade de feijão azuki (Vigna angularis). Agrarian, v.6, n.19, p.29-35, 2013.

LABOURIAU, L.G. A germinação das sementes. Organização dos Estados Americanos. Programa Regional de Desenvolvimento Científico e Tecnológico. Série de Biologia: Monografia 24, 1983.

LABOURIAU, L.G.; VALADARES, M.E.B. On the germination of seeds of Calotropis procera (Ait.) Ait. F. Anais da Academia Brasileira de Ciências, v.48, p.263-284, 1976.

MATA, J.F.; PEREIRA, J.C.S.; CHAGAS, J.F.R.; VIEIRA, L.M. Germinação e emergência de milho híbrido sob doses de esterco bovino. Amazônia: Ciência e Desenvolvimento, Belém, v.6, n.12, p.31-40, 2011.

OLIVEIRA, A.P.; ARAÚJO, J.S.; ALVES, E.U.; NORONHA, M.A.S.; CASSIMIRO, C. M.; MENDONÇA, F.G. Rendimento de feijão-caupi cultivado com esterco bovino e adubo mineral. Horticultura Brasileira, Brasília, v.19, n.1, p.81-84, 2001.

RANAL, M.A.; SANTANA, D.G. How and why to measure the germination process? Revista Brasileira de Botânica, v.29, p.1-11, 2006.

PEREIRA, R.F.; LIMA, A.S.; FILHO, F.C.F.M.; CAVALCANTE, S.N.; SANTOS, J.G.R.; ANDRADE, R. Produção de feijão vigna sob adubação orgânica em ambiente semiárido. Agropecuária científica no semiárido, v.9, n.2, p.27-32, 2013.

SALGADO, F.H.M.; SILVA, J.; OLIVEIRA, T.C.; BARROS, H.B; PASSOS, N.G.; FIDELIS, R.B. Eficiência de genótipos de feijoeiro em resposta à adubação nitrogenada. Pesquisa Agropecuária Tropical, Goiânia, v.42, n.4, p.368-374, 2012.

SILVA, E.S.; REIS, L.C.; FORESTI, A.C.; SOUZA, J.O.; BARBOSA, V.S.; RODRIGUES, E.T. Horticultura Orgânica: Saída para Pequenos Produtores Rurais de Gloria de Dourados-MS. Cadernos de Agroecologia, v.9, n.4, p. 1-6, 2014.

SILVA, R. P.; CASSIA, M.T.; VOLTARELLI, M. A.; COMPAGNON, A. M.; FURLANI, C.E.A. Qualidade da colheita mecanizada de feijão (Phaseolus vulgaris) em dois sistemas de preparo do solo. Ciência Agronômica, Fortaleza, v.44, n.1, p.61-69, 2013.

SIMS, J. T.; WOLF, D. C. Poultry waste management: agricultural and environmental issues. Advances in Agronomy, v.52, n.1, p. 2-72, 1994.

SOUZA, J.M.; SILVA, L.D.; PEREIRA, L.R.; REIS, E.F.; GARCIA, G.O. Qualidade fisiológica de sementes de feijão submetidas a águas residuárias em laboratório. Brazilian Journal of Biosystems Engineering, v.9, n.2, p.142-150, 2015. 
ZOCA, S.M.; PENN, C.J.; ROSOLEM, C.A.; ALVES, A.R.; NETO, L.O.; MARTINS, M.M. Coffee processing residues as a soil potassium amendment. Internacional Journal of Recycling of Organic Waste in Agricullture,v.3, p.155-165, 2014. 